\title{
Lazer e Uso de Substâncias Psicoativas na Adolescência: Possíveis Relações ${ }^{1}$
}

\author{
Elisângela Maria Machado Pratta ${ }^{2}$ \\ Universidade de São Paulo - Campus Ribeirão Preto \\ Universidade Camilo Castelo Branco - Campus Descalvado \\ Manoel Antonio dos Santos \\ Universidade de São Paulo - Campus Ribeirão Preto
}

\begin{abstract}
RESUMO - Este estudo objetivou: levantar as atividades de lazer praticadas por adolescentes usuários e não-usuários de drogas; verificar possíveis associações entre uso de drogas e lazer na adolescência. Participaram dele 568 adolescentes de ambos os sexos entre 14 e 20 anos que estavam cursando o ensino médio na cidade de São Carlos, SP. Estes responderam um questionário anônimo de auto-preenchimento. As análises envolveram: descrição da distribuição das variáveis na amostra; teste qui-quadrado; regressão logística múltipla. Segundo os resultados, as principais diferenças entre os grupos foram observadas nas atividades "freqüentar clubes/praias", "sair com amigos" e "freqüentar bares", mais freqüentes entre adolescentes usuários, enquanto "ir à Igreja/serviço religioso", "praticar esportes" e "sair com a família" foram mais freqüientes entre os não-usuários. Estes dados contribuem para a elaboração de ações preventivas ao uso de drogas, indicando a necessidade de estudos sobre opções de lazer para adolescentes na atualidade.
\end{abstract}

Palavras-chave: adolescência; uso de drogas; atividades de lazer.

\section{Leisure and the Use of Psychoactive Substances in Adolescence: Possible Relations}

\begin{abstract}
This study has the following objectives: to find out the leisure activities of adolescents who use drugs and those who don't; to verify possible associations between the use of drugs and leisure in adolescence. Five hundred and sixty nine male and female adolescents ranging from 14 to 20 years old who were attending high school in the city of São Carlos, in the state of São Paulo-Brazil, took part on this study. They answered an anonymous and self-filling questionnaire. The analyses involved: the description of the distribution of the variables in the sample; square-qui test; multiple logistic regression. According to the results, the main differences between the groups were observed in the activities going to clubs/beaches, going out with friends, going to bars, which were more frequent among the drug-addicted, whereas attending to religious services, practicing sports and going out with the family were more frequent among the non-addicted ones. These data may contribute for working out preventive actions against drug addiction, indicating the need of studies about leisure options for adolescents nowadays.
\end{abstract}

Key words: adolescence; use of drugs; leisure activities.

O uso de substâncias psicoativas não é um fenômeno exclusivo da época em que vivemos, uma vez que este não é um evento novo no repertório humano (Toscano Jr., 2001) e sim uma prática milenar e universal (Tavares, Béria \& Lima, 2001), com a qual o homem sempre conviveu sem que isso

1 Este trabalho recebeu apoio financeiro da CAPES e foi derivado da Dissertação de Mestrado intitulada: "Adolescência, drogadição e família: caracterização do padrão de consumo de substâncias psicoativas e avaliação da percepção dos pais em adolescentes do ensino médio" defendida na Faculdade de Filosofia, Ciências e Letras de Ribeirão Preto - Universidade de São Paulo. A realização da presente pesquisa contou com a participação voluntária de escolas e adolescentes do ensino médio da cidade de São Carlos. A análise estatística realizada foi orientada e desenvolvida, em partes, por Benedito Galvão Benze, docente do Departamento de Estatística da Universidade Federal de São Carlos e pelo aluno do curso de Estatística desta mesma instituição, Rogério Ramos.

2 Endereço: Rua Episcopal, 2474, apto 154 A, Edifício Villagio di Napoli, Vila Lutfalla II, São Carlos, SP, Brasil 13560-580.E- mail: emmppsic@ scl.terra.com.br fosse motivo de alarme social (Sanceverino \& Abreu, 2004). Entretanto este fenômeno tornou-se preocupante tanto para a comunidade científica quanto para a sociedade em geral a partir das últimas décadas do século XX, uma vez que o consumo de drogas entre os adolescentes, desde este período, tem apresentado altas prevalências e tem sido cada vez mais precoce (Guimarães, Godinho, Cruz, Kappann \& Tosta Junior, 2004; Ribeiro, Pergher \& Torossian, 1998; Toscano Jr, 2001) sendo observados graves problemas associados ao uso e abuso de drogas pelos mesmos (Kessler \& cols., 2003).

A literatura recente da área aponta que o primeiro contato com a droga geralmente ocorre na adolescência, etapa do ciclo evolutivo marcada por muitas e profundas mudanças tanto físicas quanto psíquicas, as quais tornam o adolescente mais vulnerável do ponto de vista psicológico e social (Antón, 2000; De Micheli \& Formigoni, 2000; Kalina, Kovadloff, Roig, Serran \& Cesaram, 1999; Rebolledo, Medina \& Pillon, 2004; Rivas, Rodriguez \& Gómez, 1999; Rodríguez \& Luis, 2004; Schenker \& Minayo, 2005; Silva \& Mattos, 2004; Soldera, Dalgalarrondo, Corrêa Filho \& Silva; 2004; 
Tavares \& cols., 2001). Além disso, esta fase é considerada como crucial na formação de hábitos de conduta e de modelos de socialização (Rebolledo \& cols., 2004), podendo ocorrer nela o estabelecimento de padrões estáveis de comportamento, fato que também pode levar ao consumo de drogas e ao aparecimento precoce de problemas referentes à saúde e ao ajuste social, aspectos estes que podem afetar a vida do indivíduo na idade adulta (Rivas \& cols, 1999).

Assim, por viverem um corpo e uma mente em constante transformação, fato que provoca um maior ou menor sofrimento psíquico, os adolescentes constituem um grupo de risco em relação ao consumo de drogas (Medina, Santos \& Almeida Filho, 2001; Rebolledo \& cols., 2004; Sanchéz \& Ferriani, 2004; Suárez \& Galera, 2004).

Frente a esta realidade, o consumo de drogas na adolescência tem se constituído como um grave problema social e de saúde pública, tanto na realidade brasileira quanto em outros países (Rodríguez \& Luis, 2004; Sanceverino \& Abreu, 2004; Tavares, Béria \& Lima, 2004).

Apesar dos adolescentes serem encarados como um grupo de risco, no que diz respeito ao uso de substâncias psicoativas, a literatura, de uma forma geral, aponta que os fatores que podem levar os mesmos a utilizarem drogas são variados, fato que caracteriza o fenômeno da drogadição como multideterminado. Os principais deles estão relacionados às características individuais e sociais, incluindo nestas a sociedade como um todo, a família e o grupo de pares (Outeiral, 1994).

Em função deste contexto, a prevalência e os fatores relacionados ao uso de drogas têm sido objeto de numerosos estudos. Em todo mundo, inquéritos epidemiológicos têm sido realizados com esse objetivo (Cardenal \& Adell, 1995; Miller, 1997; Miller \& Plant, 1996; Pecci, 1995; Valois, Dunham, Jackson \& Waller, 1999; Webb, Ashton, Kelly \& Kamali, 1996). No contexto brasileiro já dispomos de um número significativo de estudos epidemiológicos nessa área (Fioroni \& cols., 2003; Galduróz, Noto \& Carlini, 1997; Guimarães \& cols., 2004; Muza, Bettiol, Muccillo \& Barbieri, 1997; Sanceverino \& Abreu, 2004; Soldera \& cols, 2004; Tavares \& cols., 2001), cujos dados levantados vão ao encontro dos que foram verificados em outros inquéritos em diversas regiões do planeta, evidenciando tanto o uso de drogas lícitas quanto o de drogas ilícitas. Os resultados, no geral, têm mostrado a alta prevalência do uso de drogas lícitas (álcool e tabaco) entre estudantes do primeiro e do segundo graus e entre estudantes universitários (Medina, Santos \& Almeida Filho, 2001; Noto, 2004). Entre as drogas ilícitas a mais utilizada no Brasil é a maconha (Noto, 2004).

Segundo Schenker e Minayo (2005) os fatores de risco e de proteção em relação ao uso de drogas estão relacionados a seis domínios da vida (o individual, o familiar, o escolar, o midiático, os amigos e a comunidade de convivência) relacionados entre si, sendo que cada pesquisa na área enfatiza determinadas variáveis (por exemplo, sexo, idade, nível sócio-econômico, desempenho escolar, trabalho, uso de drogas na família etc.).

Entre os fatores de risco e proteção descritos na literatura da área interessa ao presente estudo a variável "lazer". O lazer é considerado como algo relevante para a vida das pessoas, estando diretamente ligado a uma questão de saúde
(Pereira \& Bueno, 1997) e ocorrendo em um tempo específico marcado pela ausência de um certo tipo de obrigações e deveres, sejam estes profissionais, familiares, escolares ou outros (Melo \& Alves Junior, 2003; Peres, Bodstein, Ramos \& Marcondes, 2005).

Pode-se dizer, então, que o lazer envolve um conjunto de atividades que o indivíduo realiza com prazer visando à satisfação pessoal, o descanso, o divertimento (distração, recreação, entretenimento), a participação social voluntária, o desenvolvimento geral e/ou da capacidade criadora, a formação desinteressada, expressando, assim, a cultura de um grupo ou sociedade (Camargo \& Bueno, 2003; Camargo, 1999; Dumazedier, 2001; Melo \& Alves Junior, 2003; Pereira \& Bueno, 1997).

Os interesses e as atividades praticadas pelos indivíduos na adolescência sofrem alterações no que diz respeito aos momentos de lazer. Nesta etapa o adolescente quer sair sozinho com os amigos, freqüentar lugares diferentes, ter horários diversificados para praticar atividades. Assim, na última década, tem sido dada grande importância às atividades exercidas pelos adolescentes (OMS, 1995), uma vez que foram observadas relações entre elas e diversas doenças (a curto ou a longo prazo), comportamentos de risco (como por exemplo, o uso de drogas) e comportamentos nocivos à saúde do próprio indivíduo ou de outra pessoa (Barros, Coscarelli, Coutinho \& Fonseca, 2002). Ressalta-se que os adolescentes, no tempo de lazer, costumam realizar atividades sozinhos, com amigos ou com pessoas da família, tanto no âmbito familiar quanto em outros ambientes.

Em relação ao uso do tempo livre pelos adolescentes e o consumo de substâncias psicoativas, um estudo realizado por Barria, Queiroz, Nicastri e Andrade (2000) verificou que as atividades nas horas livres por usuários de tabaco tiveram um padrão diferente em relação aos não-usuários (p 0,001). Entre os usuários, $67,5 \%$ referiram atividades realizadas fora de casa, contra 55,4\% dos não-usuários. É interessante notar que a prática de esportes aparece com porcentagens semelhantes nestes dois grupos.

Por outro lado, o estudo realizado por Robles ${ }^{3}$ citado em Jano (On-line y agenciais, 2001), verificou que o consumo de álcool entre os adolescentes espanhóis ocorre geralmente nos finais de semana, período no qual o adolescente tem maior disponibilidade para realizar atividades de lazer. Além disso, Rebolledo e cols (2004) evidenciam que o baixo envolvimento em atividades extra-curriculares está associado ao uso de substâncias psicoativas lícitas e ilícitas.

Seguindo esta reflexão, Carvalho e Carlini-Cotrim (1992) pontuam que oferecer alternativas de lazer aos adolescentes é uma estratégia relevante em relação ao uso de substâncias psicoativas, uma vez que as atividades físicas e a participação em atividades extra-curriculares são consideradas como recursos importantes neste contexto. Nesta mesma linha Cartana, Santos, Fenili \& Spricigo (2004) apontam que modos atrativos de ocupar o tempo livre podem funcionar como fatores de proteção na adolescência.

3 Dados da última pesquisa escolar do Plano Nacional sobre drogas (Espanha). 
Sendo assim torna-se importante discutir sobre quais são os tipos de atividades de lazer efetuadas pelos adolescentes, buscando verificar as possíveis associações entre estas e o uso de substâncias psicoativas. A compreensão destes aspectos apresenta uma contribuição direta no planejamento de estratégias preventivas que propiciem crescimento pessoal e educação para a saúde. Tomando por base estes elementos o presente estudo teve como objetivos: a) levantar as principais atividades de lazer praticadas por adolescentes usuários e não-usuários de substâncias psicoativas; b) verificar possíveis associações entre o uso de substâncias psicoativas e o tipo de atividade de lazer efetuada pelos adolescentes.

\section{Método}

A população-alvo da pesquisa foram os adolescentes entre 14 e 20 anos que estavam cursando o ensino médio em escolas da rede pública e privada de ensino na cidade de São Carlos - SP. Em função do número elevado de adolescentes dentro do perfil estabelecido foi selecionada uma amostra para a realização do estudo. Para a seleção adotou-se o método de amostragem probabilística por conglomerados (escolas) e estratificada (considerando-se diferentes regiões da cidade, definidas por certas características sócio-econômicas, dentro das quais estavam localizadas as escolas).

Assim, a partir das listagens de escolas fornecidas pela diretoria de ensino, a amostra de adolescentes que participou do estudo foi obtida em dois estágios: no primeiro estágio foram sorteadas as escolas e no segundo foram levantadas as turmas destas escolas que participariam da pesquisa. Foram feitas visitas às instituições escolares para entrar em contato com os alunos das turmas sorteadas, solicitar a participação dos mesmos no estudo em questão e encaminhar o Termo de Consentimento Livre e Esclarecido a ser entregue aos pais.

Responderam ao instrumento de coleta de dados 620 adolescentes sendo que destes 52 foram excluídos da amostra final por dois principais motivos: a) preenchimento incompleto ou não compreensão das perguntas; b) idade superior ao limite etário definido para a pesquisa (20 anos). Assim, participaram deste estudo 568 adolescentes que estavam cursando o ensino médio nas escolas e nas turmas previamente sorteadas, devidamente autorizados pelos pais ou responsáveis, os quais assinaram um Termo de Consentimento Livre e Esclarecido. Duzentos e cinqüenta e seis adolescentes (45,1\% da mostra) pertencem ao sexo masculino, 312 (59,9\% da mesma) são do sexo feminino e a distribuição etária é a seguinte: $5,4 \%$ das pessoas entrevistadas têm 14 anos, $25,9 \%$ têm $15,32 \%$ têm $16,23,3 \%$ têm $17,8,3 \%$ têm $18,3,5 \%$ têm 19 e $1,6 \%$ têm 20 anos. Além disso, 221 adolescentes $(38,9 \%)$ estavam cursando o primeiro ano do ensino médio, $215(37,8 \%)$ estavam no segundo ano e, por fim, $132(23,2 \%)$ estavam freqüentando o terceiro ano deste ciclo.

Foi utilizado para a coleta de dados um questionário anônimo e de auto-preenchimento composto por 62 questões fechadas, abordando dados demográficos referentes ao participante e à organização familiar do mesmo (nível de escolaridade, situação econômica, etc.), além de questões que avaliavam o nível de conhecimento, as atividades de lazer e as opiniões dos adolescentes sobre o uso de drogas e o padrão de consumo de substâncias psicoativas por parte dos mesmos. Ressalta-se que para dados a respeito do uso de drogas foi utilizado o modelo de instrumento proposto pela OMS e adaptado para a realidade brasileira por CarliniCotrim e Barbosa (1993) e para os dados sobre as atividades de lazer utilizou-se o questionário desenvolvido por Cursino (1999). O instrumento foi previamente testado em um estudo piloto com a finalidade de corrigir as imperfeições e testar o procedimento de coleta de dados.

A coleta foi realizada apenas pela pesquisadora responsável pelo projeto. Os questionários foram aplicados coletivamente em sala de aula, sem a presença do professor, em dias previamente marcados com a escola, os professores e os alunos. Quando necessário foram realizadas até duas revisitas à escola com a finalidade de aplicar os instrumentos com os alunos ausentes diminuindo, assim, o índice de perdas por faltas às aulas. Destaca-se que mesmo em posse da autorização dos pais a participação do adolescente na pesquisa foi voluntária e o mesmo estava ciente de que poderia deixar de responder ao instrumento a qualquer momento. Além disso os adolescentes foram esclarecidos sobre a contribuição dos mesmos e, frente à aceitação, eles também assinaram um Termo de Consentimento Livre e Esclarecido indicando que estavam dispostos a colaborar com o estudo em questão.

Foram poucos os casos tanto dos pais que não autorizaram a participação dos filhos adolescentes no estudo quanto dos estudantes que não quiseram participar do mesmo. Sendo assim, de uma média de 35 alunos por sala aproximadamente um ou dois alunos não foram autorizados pelos pais, o que equivale a $3 \%$ ou $6 \%$ do total de alunos da classe. Por outro lado, um número variando entre dois e três alunos por sala optou por não participar do estudo (aproximadamente 6\% a $8,6 \%$ dos mesmos). E importante ressaltar que esta pesquisa foi aprovada pelo Comitê de Ética em Pesquisa da Faculdade de Filosofia, Ciências e Letras de Ribeirão Preto da Universidade de São Paulo.

O tempo médio de aplicação do instrumento foi de 50 minutos, sendo que ao término do preenchimento os adolescentes depositavam o questionário em uma urna lacrada, deixada na frente da sala, com a finalidade de reforçar a questão do sigilo.

A análise dos dados obtidos foi efetuada com o auxílio do programa estatístico SPSS (Statistical Package for the Social Sciences). Primeiramente foram realizadas as análises descritivas, as quais envolveram: a) descrição da distribuição das variáveis na amostra estudada; b) teste do Qui-quadrado. Posteriormente foi aplicado o método da regressão logística múltipla.

\section{Resultados}

A Tabela 1 apresenta um levantamento das principais atividades de lazer que os adolescentes usuários e nãousuários de substâncias psicoativas que participaram do estudo costumam realizar no tempo livre. Neste caso, foram considerados como usuários aqueles adolescentes que já experimentaram ou fazem uso de substâncias psicoativas, exceto álcool e tabaco $(n=134)$, sem prescrição médica, e como não-usuários os adolescentes que nunca utilizaram nenhum tipo de substância psicoativa $(n=57)$.

Observando-se os resultados é possível constatar que as atividades de lazer verificadas são diversificadas, podendo 
Tabela 1. Levantamento das atividades dos adolescentes durante o tempo livre.

\begin{tabular}{|c|c|c|c|c|}
\hline \multirow[b]{2}{*}{ Atividades no tempo livre } & \multicolumn{2}{|c|}{ Usuário* $(n=134)$} & \multicolumn{2}{|c|}{ Não Usuário** $(n=57)$} \\
\hline & Freqüência Absoluta & $\begin{array}{l}\text { Frequiência } \\
\text { Relativa }(\%)\end{array}$ & Freqüência Absoluta & $\begin{array}{l}\text { Frequiência } \\
\text { Relativa(\%) }\end{array}$ \\
\hline Ir à Igreja ou serviço religioso & 22 & 16,4 & 17 & $29,8^{+}$ \\
\hline Freqüentar clubes/praias & 50 & 37,3 & 7 & $12,3^{+}$ \\
\hline Sair com amigos do mesmo sexo & 78 & 58,2 & 17 & $29,8^{+}$ \\
\hline Sair com amigos do sexo oposto & 69 & 51,5 & 12 & $21,1^{+}$ \\
\hline Praticar esportes & 55 & 41 & 27 & $47,4^{+}$ \\
\hline Assistir televisão & 85 & 63 & 33 & 57,9 \\
\hline Sair com a família & 46 & 34,3 & 23 & $40,4^{+}$ \\
\hline Sair sem destino certo & 29 & 21,6 & 6 & 10,5 \\
\hline Ir ao cinema & 39 & 29,1 & 12 & 21,1 \\
\hline Freqüentar bares & 40 & 29,8 & 2 & $3,5^{+}$ \\
\hline Ler livros & 22 & 16,4 & 13 & $22,8^{+}$ \\
\hline Ler jornais e revistas & 25 & 18,6 & 18 & $31,6^{+}$ \\
\hline Viajar & 36 & 26,9 & 11 & 19,3 \\
\hline Trabalhar em um hobby em casa & 12 & 8,9 & 2 & 3,5 \\
\hline Ficar com o namorado(a) & 56 & 41,8 & 9 & 15,8 \\
\hline Ouvir música & 99 & 73,9 & 33 & 57,9 \\
\hline Ir ao shopping & 58 & 43,3 & 18 & 31,6 \\
\hline Dormir & 2 & 1,5 & 0 & 0 \\
\hline Internet/computador & 5 & 3,7 & 1 & 1,8 \\
\hline Sair para dançar & 2 & 1,2 & 0 & 0 \\
\hline Tocar um instrumento & 2 & 3,7 & 0 & 0 \\
\hline Outros & 4 & 3 & 2 & 3,5 \\
\hline
\end{tabular}

* Foram inseridos nesta categoria os adolescente que pontuaram ter utilizado (pelo menos uma vez na vida) ou utilizar qualquer tipo de substância psicoativa (exceto álcool e tabaco).

** Foram inseridos nesta categoria os adolescentes que afirmaram nunca ter utilizado nenhum tipo de substância psicoativa; ${ }^{+}$Diferenças estatisticamente significantes entre os grupos de adolescentes (usuários e não-usuários) (teste do Qui-quadrado).

ser alocadas em quatro categorias: atividades recreativas em grupo, atividades realizadas quando sozinhos em casa, atividades com a família e, por fim, atividades culturais. Ressalta-se ainda que nesta questão o número de respostas é superior ao número de respondentes, pois cada um poderia assinalar mais de uma resposta.

Considerando-se o grupo de adolescentes usuários, as atividades que apresentaram as maiores freqüências podem ser inseridas em duas categorias: atividades realizadas quando sozinhos em casa, na qual se destacaram "ouvir música" $(73,9 \%)$ e "assistir televisão" (63\%), e atividades recreativas em grupo, como "sair com amigos do mesmo sexo" $(58,2 \%)$ e "sair com amigos do sexo oposto" (51,5\%).

Por outro lado, no que diz respeito ao grupo de adolescentes não-usuários constatou-se que as atividades mais frequientes se inserem em três categorias principais. Primeiramente as atividades realizadas quando sozinhos em casa, na qual se destacaram "ouvir música" e "assistir televisão", ambas com $57,9 \%$ de indicações. Posteriormente a categoria atividades recreativas em grupo, na qual se destaca a prática de esportes $(47,4 \%)$ e a categoria atividades em família, na qual "sair com a família" obteve $40,4 \%$ de indicações.

Comparando-se os dois grupos de adolescentes foram verificadas algumas diferenças estatisticamente significativas entre eles. Nas atividades "ir à Igreja ou serviço religioso" e "freqüentar clubes/praias", atividades que se inserem na categoria atividades recreativas em grupo, foram verificadas diferenças entre os grupos de adolescentes (usuários e nãousuários) apesar destas categorias não terem demonstrado freqüências elevadas para os mesmos. No que diz respeito à atividade "ir à Igreja ou serviço religioso" constata-se que o percentual de não-usuários que afirmaram realizar esta atividade no tempo livre $(29,8 \%)$ é significativamente maior que o mesmo percentual observado no grupo de usuários $(16,4 \%)(p<0,05)$.

Em relação à atividade "freqüentar clubes/praias", esta apresentou uma freqüência significantemente mais elevada no grupo de usuários quando comparada ao percentual obtido no grupo de adolescentes não-usuários $(37,3 \%$ e $12,3 \%$ respectivamente $)(p<0,05)$.

Uma outra diferença constatada entre os grupos refere-se às atividades "sair com amigos do mesmo sexo" e "sair com amigos do sexo oposto", as quais se inserem na categoria atividades recreativas em grupo. Para ambas atividades foi observado um percentual significantemente maior para o grupo de usuários $(58,2 \%$ e $51,5 \%)$ comparativamente ao obtido pelo grupo de não-usuários nestas mesmas atividades $(29,8 \%$ e $21,1 \%)(p<0,01)$. Isso quer dizer que os adolescentes do grupo de usuários saem mais com os amigos, tanto do mesmo sexo quanto do sexo oposto, no tempo livre do que os adolescentes do grupo de não-usuários.

Por outro lado, as atividades "praticar esportes" e "sair com a família" apresentaram porcentagens significantemente maiores para o grupo de adolescentes não-usuários $(47,4 \%$ e $40,4 \%)$ quando comparadas às porcentagens obtidas pelos adolescentes do grupo de usuários $(41 \%$ e $34,3 \%$ respectivamente) $(p<0,01)$. Ou seja, os adolescentes do grupo de não-usuários costumam realizar mais estas atividades no tempo livre do que o grupo de adolescentes usuários.

Foram ainda constatadas diferenças estatisticamente significativas entre os grupos no que se refere às atividades: 
Tabela 2. Atividades de lazer realizadas pelos adolescentes segundo uso na vida de álcool e tabaco.

\begin{tabular}{|c|c|c|c|c|}
\hline \multirow[t]{2}{*}{ Atividades de lazer } & \multicolumn{2}{|c|}{$\begin{array}{c}\text { Álcool } \\
(n=412)\end{array}$} & \multicolumn{2}{|c|}{$\begin{array}{c}\text { Tabaco } \\
(n=149)\end{array}$} \\
\hline & FA & $\operatorname{FR}(\%)$ & FA & $\operatorname{FR}(\%)$ \\
\hline Ir à Igreja ou serviço religioso & 86 & 20,9 & 20 & 13,4 \\
\hline Freqüentar clubes/praias & 124 & 30,1 & 58 & 38,9 \\
\hline Sair com amigos do mesmo sexo & 228 & 55,3 & 101 & 67,8 \\
\hline Sair com amigos do sexo oposto & 198 & 48,1 & 96 & 64,4 \\
\hline Praticar esportes & 189 & 45,9 & 58 & 38,9 \\
\hline Assistir TV & 286 & 69,4 & 96 & 64,4 \\
\hline Sair com a família & 149 & 36,2 & 53 & 35,6 \\
\hline Sair sem destino & 62 & 15 & 32 & 21,5 \\
\hline Ir ao cinema & 145 & 35,2 & 52 & 34,9 \\
\hline Freqüentar bares & 85 & 20,6 & 50 & 33,6 \\
\hline Ler livros & 78 & 18,9 & 26 & 17,4 \\
\hline Ler jornais/ revistas & 90 & 21,8 & 32 & 21,5 \\
\hline Viajar & 93 & 22,6 & 42 & 28,2 \\
\hline Trabalhar em um hobby em casa & 31 & 7,5 & 12 & 8,1 \\
\hline Ficar com o namorado(a) & 141 & 34,2 & 60 & 40,3 \\
\hline Ouvir música & 311 & 75,5 & 118 & 79,2 \\
\hline Ir ao shopping & 194 & 47,1 & 71 & 47,7 \\
\hline Outros & 38 & 9,2 & 14 & 9,4 \\
\hline
\end{tabular}

"freqüentar bares", "ler livros" e "ler jornais e revistas". No caso da atividade "freqüentar bares", a qual se encaixa na categoria atividades recreativas em grupo, os dados obtidos revelam que o percentual verificado no grupo de adolescentes usuários foi significantemente maior do que o que foi observado para o grupo de adolescentes não-usuários ( $29,8 \%$ e $3,5 \%$ respectivamente) $(p<0,01)$.

Por fim, quanto às atividades "ler livros" e "ler jornais e revistas", as quais podem ser inseridas nas atividades realizadas quando sozinhos em casa, nota-se que as porcentagens apresentadas para o grupo de adolescentes não-usuários foram significantemente maiores $(22,8 \%$ e $31,6 \%$ respectivamente) que as observadas no grupo de adolescentes usuários $(16,4 \%$ e $18,6 \%)(p<0,05)$.

Para as demais atividades abordadas não foram verificadas diferenças estatisticamente significativas entre os grupos de adolescentes. A Tabela 2 abaixo traz as principais atividades realizadas por adolescentes que já fizeram uso na vida de álcool e tabaco.

Verificando-se os dados descritos na Tabela 2 alguns pontos necessitam ser ressaltados. No que se refere ao grupo de adolescentes que afirmou utilizar álcool na vida $(n=412)$ constata-se que a principal atividade de lazer realizada pelos mesmos é "ouvir música" (75,5\% de indicações), sendo esta seguida pela atividade "assistir televisão" $(69,4 \%)$. Destaca-se ainda que 55,3\% dos adolescentes deste grupo afirmaram "sair com amigos do mesmo sexo" no tempo que têm livre para o lazer.

Por outro lado, os adolescentes que pontuaram ter feito uso na vida de tabaco apresentaram como principais atividades no tempo livre as seguintes: "ouvir música" (79,2\%), "sair com amigos do mesmo sexo" (67,8\%), "sair com amigos do sexo oposto" e "assistir televisão", ambas com $64,4 \%$ de indicações.

Com a finalidade de compreender melhor as possíveis associações entre o uso de substâncias psicoativas e o tipo de atividade de lazer praticada pelos adolescentes, foram realizadas análises mais apuradas por meio da regressão logística, priorizando-se, neste caso, o uso na vida de álcool e tabaco, substâncias estas que foram as mais consumidas pelos adolescentes desta amostra.

No que diz respeito ao uso na vida de álcool ( $p=0,000 \mathrm{e}$ $R^{2}=16,3 \%$.) pelos adolescentes, as variáveis estatisticamente significativas no modelo logístico binário, concernentes às atividades realizadas no tempo livre, foram: AMISEXOP ( sair com amigos do sexo oposto) $(p=0,007)$; TV $(p=0,012)$; BARES (freqüentar bares) $(p=0,035)$; NAMORAR $(p=0,004)$.

Tabela 3. Levantamento dos fatores associados ao uso na vida de álcool entre adolescentes do ensino médio da cidade de São Carlos.

\begin{tabular}{lccc}
\hline Fatores associados & Grupos & p valor & RP* 95\%** \\
\hline $\begin{array}{l}\text { Sair com amigos do sexo oposto } \\
\text { (AMISEXOP) }\end{array}$ & $1 \times 0$ & 0,007 & 2,16 \\
Assistir TV & $1 \times 0$ & 0,012 & $1,23-3,79$ \\
Freqüentar bares & $1 \times 0$ & 0,035 & 2,33 \\
Namorar & $1 \times 0$ & 0,004 & 2,12 \\
\hline
\end{tabular}

* Razões de chances (medem o risco de um determinado grupo usar ou não certa substância).

** Intervalo de confiança. 
Os dados descritos na Tabela 3 revelam que o grupo de adolescentes que sai com amigos do sexo oposto (1) apresentou 2,16 (IC 95\% 1,23; 3,79) vezes mais chances de fazer uso na vida de álcool do que o grupo de adolescentes que não costumam sair.

Um outro dado interessante diz respeito à atividade "assistir TV". Verificando-se os resultados nota-se que o grupo que afirmou assistir TV no tempo livre apresentou, 1,88 vezes mais chance (IC 95\% 1,15; 3,07) de fazer uso de álcool na vida do que o grupo que não assiste.

Ainda no que concerne à probabilidade de fazer uso de álcool na vida, os dados mostram que o grupo de adolescentes que costuma freqüentar bares nos momentos de lazer apresentou 2,33 vezes mais chances (IC 95\% 1.06; 5,12) que o grupo daqueles que não freqüentam. Já o grupo de adolescentes que namoram apresentou 2,12 vezes mais chances (IC 95\% 1,26; $3,55)$ que o grupo daqueles que não namoram.

No que diz respeito à variável dependente uso na vida de tabaco $\left(p=0,000\right.$ e $\left.R^{2}=22,6 \%\right)$, as variáveis estatisticamente significativas no modelo logístico binário, considerando-se as atividades realizadas pelos adolescentes no tempo livre, foram duas: AMISEXOP (sair com amigos do sexo oposto) ( $p=0,001)$ e ESPORTES (praticar esportes) ( $p=0,009)$. Sendo assim, pode-se dizer que o grupo que costuma sair com amigos do sexo oposto nos momentos de lazer e o grupo de adolescentes que afirmaram não praticar esportes apresentaram maiores chances de fazer uso na vida de tabaco do que aqueles que não saem com amigos do sexo oposto e que praticam esportes $(2,47, I C 95 \% 1,43 ; 4,27$ e $1,83, I C 96 \%$ 1,$16 ; 2,87$ respectivamente). Novamente, como no caso do uso na vida de álcool, a influência do grupo de amigos aparece como um fator de risco também no que diz respeito ao uso na vida de tabaco.

\section{Discussão}

Refletindo-se sobre os dados descritos, nota-se que, de uma forma geral, as principais atividades praticadas pelos adolescentes envolvem aquelas que podem ser realizadas dentro de casa e aquelas que são realizadas fora de casa. Em relação às atividades realizadas fora de casa, o grupo de adolescentes usuários destacou, respectivamente, "sair com os amigos do mesmo sexo" e "sair com amigos do sexo oposto", enquanto para o grupo de adolescentes não-usuários o destaque foi para as atividades "praticar esportes" e "sair com a família".

Em relação ao uso do tempo livre pelos adolescentes, um estudo realizado por Barria e cols. (2000) verificou que, entre os usuários de tabaco, foco do estudo realizado pelos autores, 67,5\% referiram atividades fora de casa (sair/viajar com amigos/namorado(a); atividades culturais como cinema, teatro e shows; frequientar bares e festas), contra 55,4\% dos não-usuários. A prática de esportes aparece com porcentagens semelhantes nos dois grupos.

Entretanto, é importante pontuar que, no caso do presente estudo, no que diz respeito à prática de esportes, os dados obtidos apresentaram diferenças significativas entre os grupos, uma vez que as porcentagens verificadas foram maiores para o grupo de não-usuários do que para o grupo de usuários. Porém é importante ressaltar que outros estudos realizados na realidade brasileira não encontraram associação entre o uso de drogas e a prática de atividade física regular como o realizado por Tavares e cols. (2004) e por Carvalho e Carlini-Cotrim (1992).

Apesar disso, este é um dado interessante por retratar a importância da atividade esportiva no cotidiano do adolescente, uma vez que o fato do indivíduo realizar algum tipo de atividade esportiva pode levá-lo a apresentar uma maior preocupação com a saúde e isso pode contribuir para a não utilização de substâncias psicoativas. Assim, o incentivo ao esporte, desde cedo, deve ser uma preocupação constante uma vez que a prática de atividades esportivas pode funcionar como um fator de proteção em relação ao uso de substâncias psicoativas. Além disso, este achado chama a atenção para a avaliação das possibilidades de prática de esportes oferecidas ao indivíduo na cidade em que reside. Entretanto estes aspectos necessitam ser explorados em pesquisas futuras, avaliando, por exemplo, indivíduos que praticam e não praticam esportes bem como o tipo de atividade esportiva realizada e o uso e não-uso de substâncias psicoativas.

Por outro lado, frequientar bares foi uma atividade de porcentagem significativamente maior para o grupo de usuários quando comparada ao grupo de não-usuários. Uma hipótese que pode ser levantada em relação a esta questão refere-se diretamente às opções de lazer disponíveis para os adolescentes em uma cidade de médio porte do interior paulista. Apesar de existirem opções culturais de lazer, em lugares diversificados, muitas vezes os adolescentes preferem, quando saem com os amigos, frequientar outros lugares que consideram de maior "agitação". Os lugares mais comuns que eles costumam freqüentar são barzinhos, danceterias, shopping e clubes. Em muitos destes ambientes constata-se a presença de substâncias psicoativas, principalmente as legalizadas. Desta maneira, muitos adolescentes, acompanhados de amigos, podem entrar em contato com tais substâncias nestes lugares (Rebello, Monteiro \& Vargas, 2001) e, se o amigo utiliza alguma substância, as chances do adolescente experimentar ou começar a utilizar podem ser maiores, embora isso dependa também de suas habilidades para lidar com as pressões exercidas pelo grupo (Rodríguez \& Luis, 2004).

Outro dado interessante de nota refere-se à atividade "ir à Igreja ou serviço religioso". Considerando-se o grupo de usuários verifica-se que esta atividade apresentou freqüências menores se comparadas ao grupo de adolescentes não-usuários. Neste sentido, alguns estudos afirmam que o envolvimento com a religião pode estar associado ao uso ou não-uso de substâncias psicoativas, embora este dado ainda apresente controvérsias na literatura da área (Soldera \& Dalgalorrondo, 1999).

Miller e Plant (1996) apontam que a ausência de religião está associada a um maior uso de drogas pelos estudantes, enquanto jovens que possuem algum tipo de vínculo religioso apresentam um menor uso de substâncias psicoativas. Estes dados caminham na direção dos achados de Soldera e cols (2004), que evidenciam que estudantes com educação religiosa na infância apresentam menor uso pesado de drogas, e também se aproxima dos dados obtidos por Tavares e cols. (2004), que pontuam que o fato do adolescente praticar a religião está associado a um menor uso de drogas. 
Quanto à atividade "sair com a família", os adolescentes não-usuários costumam realizar mais esta atividade do que aqueles que fazem uso de substâncias psicoativas. Este dado pode indicar a existência de um bom vínculo ou de uma boa interação com os pais ou ainda a preocupação em compartilhar atividades e momentos entre pais e filhos, aspectos estes que, segundo Schenker e Minayo (2005), funcionam como fatores de proteção contra o uso de drogas.

Em relação aos dados obtidos por meio da regressão logística, estes trazem à tona a reflexão sobre alguns elementos importantes. No que diz respeito ao uso na vida de álcool, os achados do presente estudo solicitam uma reflexão sobre a influência do grupo de amigos, a presença da mídia na vida dos adolescentes, a freqüência a bares e a questão do namoro nesta etapa da vida. Isso porque, em linhas gerais, os achados mostram que adolescentes que costumam sair com amigos, ver televisão, freqüentar bares e namorar no tempo livre apresentaram maiores chances de fazer uso de álcool na vida. Por outro lado, em relação ao tabaco, os dados apontam que adolescentes que costumam sair com amigos no tempo livre e não praticar esportes têm maiores chances de fazer uso na vida deste.

Pode-se refletir sobre a questão da influência dos pares, no sentido em que o adolescente que costuma sair com amigos que utilizam, por exemplo, álcool ou tabaco, pode começar a utilizar esta substância por pressão do próprio grupo e para se sentir integrado a ele, uma vez que os amigos podem ser considerados como modelos de comportamento (Hoffmann \& Cerbone, 2002).

Em relação ao tabaco, estudos buscando avaliar a questão do tabagismo entre adolescentes encontraram uma associação positiva entre o uso desta substância e a presença de tabagismo entre o grupo de amigos (Malcon, Menezes \& Chatkin, 2003). Desta forma, o tabagismo no grupo de amigos pode ser caracterizado como um fator de risco em relação ao uso de tabaco na adolescência, conforme estudo realizado de Malcon e cols. (2003). Além deste aspecto os autores encontraram outros três pontos que foram caracterizados como fatores de risco para o tabagismo na adolescência: idade do adolescente, escolaridade do mesmo e fumo pelos irmãos mais velhos.

Entretanto, a influência do grupo de amigos não pode ser vista de uma maneira simplista, uma vez que a afiliação a pares que aprovam ou toleram o uso de substâncias psicoativas corresponde ao final de um percurso no qual diversos fatores se combinam aumentando a probabilidade de uso abusivo. Contudo é necessário pontuar, o outro lado, pois grupos de amigos com objetivos e expectativas de realização na vida também têm papel fundamental numa etapa existencial em que as influências dos pares são cruciais (Schenker \& Minayo, 2005).

Em relação à influência da mídia no comportamento do adolescente também é importante analisar os aspectos positivos e negativos da mesma, que apresenta um papel dominante no contexto social, veiculando modelos ideais, influenciados pela ideologia atual (Ribeiro \& cols., 1998).

No caso da televisão, é possível levantar uma série de propagandas de bebidas alcoólicas, particularmente de cerveja, que são diariamente exibidas. Estes comerciais costumam ser chamativos, sendo associados a situações divertidas envolvendo jovens e lazer, além de, segundo Schenker e
Minayo (2005), vincularem o uso à imagem de artistas, ao glamour da sociabilidade e à sexualidade, retratando estas substâncias como mediadores de fama e sucesso. O uso de bebidas é veiculado, também, como uma forma de esquecer os problemas ou para relaxar (Sanceverino \& Abreu, 2004). Estas campanhas intensificam-se no período do verão, o qual coincide com o período de férias do calendário escolar. Uma das principais atividades de lazer dos adolescentes, tanto nas férias quanto no período normal de aula, é assistir televisão. Assim o adolescente, que está em busca de uma identificação, pode ser diretamente influenciado por este tipo de estímulo.

Entretanto, por si só a propaganda não tem um efeito devastador em termos de persuasão. Além disso, as informações veiculadas podem gerar uma reflexão crítica moderando o risco potencial de exposição (Schenker \& Minayo, 2005), e este veículo pode ser utilizado na prevenção primordial e para convidar os adolescentes para atividades diversificadas, de interesse dos mesmos, em ambientes externos (Barros \& cols., 2002) .

Neste sentido é importante lembrar que, no presente estudo, a atividade mais realizada pelos adolescentes é "assistir TV" e a bebida mais consumida pelos mesmos é a cerveja. Este dado aponta, então, a importância de se oferecer aos adolescentes novas opções para atividades de lazer, opções que possibilitem ao adolescente realizar algo prazeroso e que tenha uma contribuição significativa para seu desenvolvimento.

Além da questão da influência da mídia é importante lembrar que o álcool, utilizado desde a Antigüidade, está sempre presente nos momentos de festa e comemoração, sendo considerado o símbolo farmacológico da mesma. Desta forma, está sempre presente nas reuniões de amigos ou de algum grupo para comemorar algo bom que aconteceu com alguém ou simplesmente para curtir a vida (Toscano Jr., 2001).

No caso específico de "freqüentar bares" pode-se dizer que o fato do adolescente estar em um ambiente público, no qual esta substância está disponível, pode levá-lo a utilizar a mesma, uma vez que a proibição da venda de bebidas alcoólicas para menores é muitas vezes descumprida. Além disso, mesmo que exista o controle, se o adolescente está com um grupo e neste existem indivíduos mais velhos, estes podem solicitar a bebida e compartilhar com o adolescente. Sendo assim, segundo Schenker e Minayo (2005), o fácil acesso e a disponibilidade da substância, associado a outros aspectos como fatores do ambiente social e desorganização social, podem levar o indivíduo a utilizá-la.

Ainda no que concerne a relações interpessoais, o fato do adolescente "namorar" pode levá-lo ao uso de substâncias psicoativas, caso namore uma pessoa que seja usuária destas (no caso, o álcool) e ele comece a utilizá-la para acompanhar a(o) namorada(o) ou para não se sentir "careta" (considerando que a avaliação do outro tem um peso muito grande para o adolescente).

Por fim, em relação à prática de esportes e o uso na vida de tabaco, os dados demonstram que aqueles adolescentes que não praticam esportes têm maiores chances de fazer uso na vida de tabaco do que aqueles que praticam. Isso demonstra que a prática de esportes pode atuar como um fator preventivo 
em relação ao uso de tabaco. Neste sentido, Jonathan, Rivera, Muñoz \& Pérez (2000) encontraram, em um estudo com adolescentes, uma associação estatisticamente significativa entre falta de atividade esportiva e uso de tabaco pelos mesmos. Segundo estes autores, as atividades físicas poderiam ser consideradas não só como uma boa opção em termos de prevenção, mas também como uma possível opção auxiliar no tratamento do tabagismo.

\section{Considerações Finais}

O uso de substâncias psicoativas na atualidade é algo complexo que se relaciona a uma imensa gama de fatores e merece atenção diferenciada tanto por parte dos pesquisadores da área quanto pela comunidade em geral. Os dados levantados neste estudo apresentam alguns aspectos que precisam ser evidenciados quando se aborda a temática do uso de substâncias psicoativas na adolescência e suas possíveis associações com atividades de lazer realizadas pelos adolescentes.

Os dados evidenciam uma variedade considerável de atividades realizadas pelos adolescentes no momento de lazer. Entre os adolescentes usuários as atividades de lazer que apresentaram as maiores freqüências foram "ouvir música", "assistir televisão" e "sair com amigos", enquanto para o grupo de adolescentes não-usuários as atividades mais freqüentes foram "ouvir música" e "assistir televisão", "praticar esportes" e "sair com a família”. As diferenças significativas entre os dois grupos de adolescentes considerados para o estudo apareceram em atividades específicas. Assim, "ir à Igreja ou serviço religioso", "praticar esportes" e "sair com a família" foram mais freqüentes entre os adolescentes não-usuários, enquanto atividades como "freqüentar clubes/praias", "sair com amigos" e "freqüentar bares" foram pontuadas como sendo mais comumente realizadas por adolescentes usuários.

Em relação ao álcool, os achados deste estudo evidenciam que, como fatores facilitadores do uso na vida desta substância, foram identificados "assistir televisão", "sair com amigos", "namorar" e "freqüentar bares". Já em relação ao tabaco, fatores como "sair com os amigos" e "não praticar esportes" foram relacionados a uma maior probabilidade de uso desta substância na vida.

As diferenças verificadas entre os dois grupos (usuários e não-usuários) em relação ao tipo de atividade realizada pelos mesmos são informações importantes para a elaboração de programas de prevenção ao uso de substâncias psicoativas entre os adolescentes, uma vez que os dados observados levam-nos a refletir sobre as opções de lazer disponíveis para os adolescentes na atualidade. Sendo assim, há a necessidade de novos estudos buscando lançar luz sobre este aspecto. Isso porque, segundo Schenker e Minayo (2005), o incentivo à participação nas atividades escolares, da comunidade e de movimentos sociais ou de solidariedade pode funcionar como um intenso fator protetor, aspecto este que passa pelas atividades de lazer, foco do presente estudo.

Além disso, os dados obtidos no presente estudo permitem evidenciar a necessidade de uma reflexão sobre novas atividades de lazer que podem ser oferecidas aos adolescentes incentivando, principalmente, a questão esportiva e cultural, não apenas como forma de preencher o tempo do adolescente e sim como forma de oferecer oportunidades de crescimento pessoal, de excitação, desafio e alívio do tédio (Sanceverino \& Abreu, 2004) por meio de atividades que tragam prazer e que despertem o interesse do adolescente. $\mathrm{O}$ estudo de Carvalho e Carlini-Cotrim (1992) reforça esta conclusão, evidenciando que a prevenção ao uso de substâncias psicoativas pelo simples preenchimento do tempo livre parece ter pouco resultado.

Assim, estratégias de ocupação do tempo livre e a reivindicação de espaços de lazer e convivência para jovens devem estar associadas a um trabalho de educação para a saúde que contribua para um padrão de vida saudável, trazendo orientações específicas sobre hábitos alimentares, atividades esportivas e recreativas e vida sexual segura (Sanceverino \& Abreu, 2004) e que apresente, então, uma preocupação com a qualidade, em termos de vivências e de abertura de espaços para questionamentos e desenvolvimento criativo (Carvalho \& Carlini-Cotrim, 1992).

Estes achados empíricos colaboram, portanto, para a compreensão das possíveis relações entre uso de substâncias psicotivas e atividades de lazer, ressaltando alguns aspectos importantes a serem considerados no processo de prevenção visando à diminuição de comportamentos que podem trazer risco à saúde, e contribuindo para o desenvolvimento de ações preventivas que valorizem a saúde e a vida do adolescente.

\section{Referências}

Antón, D. M. (2000). Drogas: conhecer e educar para prevenir. São Paulo: Scipione.

Barría, A. C. R., Queiroz, S., Nicastri, S. \& Andrade, A. G. (2000). Comportamento do universitário da área de biológicas da Universidade de São Paulo, em relação ao uso de drogas. Revista de Psiquiatria Clínica, 27(4), 215-224.

Barros, R., Coscarelli, P., Coutinho, M. F. G. \& Fonseca, A. F. (2002). O uso do tempo livre por adolescentes em uma comunidade metropolitana no Brasil. Adolescência Latinoamericana, 3(2), 0-0. Retirado em 20/02/2003 do <http://ral-adolec.bvs.br/scielo. php?script=sci_arttext\&pid=S1414-71302002000200008\&lng= es\&nrm=iso $>$

Camargo, R. A. A. \& Bueno, S. M. V. (2003). Lazer, a vida além do trabalho para uma equipe de futebol entre trabalhadores de hospital. Revista Latino-Americana de Enfermagem, 11(4), 490-498.

Camargo, L. L. (1999). O que é lazer. São Paulo: Brasiliense.

Cardenal, C. A. \& Adell, M. N. (1995). Consumo de alcohol en escolares. Medicina Clínica, 105, 481-486.

Carlini-Cotrim, B. \& Barbosa, M. T. S. (1993). Pesquisa epidemiológicas sobre o uso de drogas entre estudantes: um manual de orientações gerais. São Paulo: CEP Medicina.

Carvalho, V. A. \& Carlini-Cotrim, B. (1992). Atividades extracurriculares e prevenção ao uso de drogas: uma questão polêmica. Revista de Saúde Pública, 26(3), 145-149.

Cartana, M. H. F., Santos, S. M. A., Fenili, R. M. \& Spricigo, J. S. (2004). Prevenção do uso de substâncias psicoativas. Texto Contexto Enfermagem, 13(2), 286-289.

Cursino, E. A. (1999). Sexualidade, Aids e Drogas: Informações, concepções e percepções de alunos e professoras de uma escola de primeiro grau. Dissertação de Mestrado, Universidade de São Paulo, Ribeirão Preto. 
De Micheli, D. \& Formigoni, M. L. O. S. (2000). Screening of drug use in a teenage brazilian sample using the drug use screening inventory (DUSI). Addictive Behaviors, 25(5), 683-691.

Dumazedier, J. (2001). Lazer e cultura popular (3 ed.). São Paulo: Perspectiva.

Fiorini, J. E., Alves, A. L., Ferreira, L. R., Fiorini, C. M., Durães, S. W., Santos, R. L. D., Nascimento, L. C., Geraldini, A. M. V. \& Ortiz, C. F. (2003). Use of licit and illicit drugs at the University of Alfenas. Revista do Hospital das Clínicas, 58(4), 199-206.

Galduróz, J. C. F., Noto, A. R. \& Carlini, E. A. (1997). IV Levantamento sobre o uso de drogas entre estudantes do $1^{\circ}$ e do $2^{\circ}$ graus em 10 capitais brasileiras. São Paulo: CEBRID.

Guimarães, J. L., Godinho, P. H., Cruz, R., Kappann, J. \& Tosta Junior, L. A. (2004). Consumo de drogas psicoativas por adolescentes escolares de Assis, SP. Revista de Saúde Pública, 38(1), 130-132.

Hoffmann, J. P. \& Cerbone, F. G. (2002). Parental substance use disorder and the risk of adolescent drug abuse: na event history analysis. Drug and Alcohol Dependence, 66, 255-264.

Jano On-Line y Agencias (2001). Según OMS, una de cada cuatro muertes de jóvenes europeos está relacionada com el alcohol. Retirado em 20/02/2003 em http://db.doyma.es/cgi-bin/wdbcgi. exe/doyma

Jonathan, J., Rivera, C., Muñoz, J. O., Pérez, E. C (2000). Depresión en la adolescencia: su relación con actividad deportiva y consumo de drogas. Revista Médica del Instituto Mexicano del Seguro Social, 38(5), 371-379.

Kalina, E., Kovadloff, S., Roig, P. M., Serran, J. C. \& Cesaram, F. (1999). Drogadição hoje: Indivíduo, família e sociedade. Porto Alegre: Editora Artes Médicas.

Kessler, F., Diemen, L., Seganfredo, A. C., Brandão, I., Saibro, P., Scheidt, B., Grillo, R. \& Ramos, S. P. (2003). Revista de Psiquiatria do Rio Grande do Sul, 25(1), 33-41.

Malcon, M. C., Menezes, A. M. B. \& Chatkin, M. (2003). Prevalência e fatores de risco para tabagismo em adolescentes. Revista de Saúde Pública, 37(1),1-7.

Medina, M. G., Santos, D. N. \& Almeida Filho, N. (2001). Epidemiologia do consumo de substâncias psicoativas. Em S. D. Seibel \& A. Toscano Jr. (Orgs.). Dependência de drogas. (pp. 161-179). São Paulo: Editora Atheneu.

Melo, V. \& Alves Junior, E. D. (2003). Introdução ao lazer. São Paulo: Manole.

Miller, P. (1997). Family structure, personality, drinking, smoking and illicit drug use: a stydy of uk teenagers. Drug Alchohol Depend, 45(1-2), 121-129.

Miller, P. M. \& Plant, M. (1996). Drinking, smoking and illicit drug use among 15 and 16 year olds in the united kingdom. British Medical Journal, 313, 394-397.

Muza, G., Bettiol, H., Muccillo, G. \& Barbieri, M. A. (1997). Consumo de substâncias psicoativas por adolescentes escolares de Ribeirão Preto, SP (Brasil). I - Prevalência do consumo por sexo, idade e tipo de substância. Revista de Saúde Pública, 31, 163-170.

Noto, A. (2004). Os índices de consumo de psicotrópicos entre adolescente no Brasil. Em I. Pinsky \& M. Bessa (Orgs). Adolescência e drogas (pp. 45-53). São Paulo: Contexto.

OMS (1995). La salud de los jóvenes: um reto y uma esperanza. Genebra: OMS.

Outeiral, J. (1994). Adolescer: Estudos sobre adolescência. Porto Alegre: Artes Médicas.
Pecci, M. C. (1995). Varones jóvenes y sustancias psicoativas. Acta Psiquiatrita y Psicologica de America Latina, 41, 288-299.

Pereira, M. E. R. \& Bueno, S. M. V. (1997). Lazer - um caminho para aliviar as tensões no ambiente de trabalho em uti: uma concepção da equipe de enfermagem. Revista LatinoAmericana de Enfermagem, 5(4), 75-83.

Peres, F. F., Bodstein, R., Ramos, C. L. \& Marcondes, W. B. (2005). Lazer, esporte e cultura na agenda local: a experiência de promoção da saúde em Manguinhos. Ciência e Saúde Coletiva, 10(3), 757-769.

Rebello, S., Monteiro, S. \& Vargas, E. P. (2001). A visão de escolares sobre drogas no uso de um jogo educativo. Interface - Comunicação, Saúde, Educação,5(8), 75-88.

Rebolledo, E. A. O., Medina, N. M. O. \& Pillon, S. C. (2004). Factores de riesgo asociados al uso de drogas em estudiantes adolescentes. Revista Latino-Americana de Enfermagem, 12, 369-375.

Ribeiro, T. W., Pergher, N. K. \& Torossian S. D. (1998). Drogas e adolescência: uma análise da ideologia presente na mídia escrita destinada ao grande público. Psicologia: Reflexão $e$ Crítica, 11(3), 421-430.

Rivas, M. J., Rodriguez, J. A. C. \& Gómez, J. L. G. (1999). Consumo de drogas en adolescentes de la Comunidad de Madrid. Adicciones, 1(4), 311-322.

Rodríguez, G. M. \& Luis, M. A. V. (2004). Estúdio descriptivo del uso de drogas em adolescentes de educación media superior de la ciudad de Monterrey, Nueva Leon, México. Revista Latino-Americana de Enfermagem. 12, 391-397.

Sanceverino, S. L. \& Abreu, J. L. C. (2004). Aspectos epidemiológicos do uso de drogas entre estudantes do ensino médio no município de Palhoça 2003. Ciência e Saúde Coletiva, 9(4), 1047-1056.

Sánchez, F. M. \& Ferriani, M. G. C. (2004). Percepción de padres e profesores de los factores de riesgo para el uso de drogas lícitas e ilícitas em los escolares, Revista Latino-Americana de Enfermagem, 12, 352-358.

Schenker, M. \& Minayo, M. C. S. (2005). Fatores de risco e de proteção para o uso de drogas na adolescência. Ciência e Saúde Coletiva, 10(3), 707-717.

Silva. V. A. \& Mattos, H. F. (2004). Os jovens são mais vulneráveis às drogas? Em I. Pinsky \& M. Bessa (Orgs), Adolescência e drogas (pp. 31-44). São Paulo: Contexto.

Soldera, M., Dalgalarrondo, P., Corrêa Filho, H. R. \& Silva, C. A. M. Uso de drogas psicotrópicas por estudantes: prevalência e fatores sociais associados. Revista de Saúde Pública, 38(2), 277-283.

Soldera, M. A. \& Dalgalarrondo, P. (1999). Religião e uso frequiente de drogas por estudantes do segundo grau. Medicina, 32(1), 9-12.

Suárez, R. E. S. \& Galera, S. A. F. (2004). Discurso de los padres sobre el uso de drogas lícitas e ilícitas percibido por estudiantes universitários. Revista Latino-Americana de Enfermagem, 12, 406-411.

Tavares, B. F., Béria, J. U. \& Lima, M. S. (2004). Fatores associados ao uso de drogas entre adolescentes escolares. Revista de Saúde Pública, 38(6), 787-796.

Tavares, B. F., Béria, J. U. \& Lima, M. S. (2001). Prevalência do uso de drogas e desempenho escolar entre adolescentes. Revista de Saúde, 35(2), 150-158. 
Toscano Jr., A. (2001). Adolescência e drogas. Em S. D. Seibel \& A. Toscano Jr. (Orgs.), Dependência de drogas (pp. 283-302). São Paulo: Editora Atheneu.

Valois, R. F., Dunham, A. C. A., Jackson, K. L. \& Waller, J. (1999). Association between employment and substance abuse behaviors among public high school adolescents. Journal Adolescent Health, 25, 256-263.
Webb, E., Ashton, C. H., Kelly, P.\& Kamali, F. (1996). Alcohol and drug use in UK university students. Lancet, 348, 922-925.

Recebido em 07.07.2005

Primeira decisão editorial em 20.04.2006

Versão final em 25.05.2006

Aceito em 26.01.2007 